\title{
Complicated Skin and Skin Structure Infections (cSSSI's): A Comprehensive Review
}

\author{
K V Ramana ${ }^{1, *}$, Venkata BharatKumar Pinnelli ${ }^{2}$, Bhanu Prakash ${ }^{3}$, Wilma Delphine Silvia $\mathrm{CR}^{4}$, Sabitha Kandi ${ }^{5}$, \\ CH V Sharada ${ }^{6}$, Anand Kalaskar ${ }^{1}$, Sanjeev D Rao ${ }^{1}$, Ratna Mani ${ }^{7}$, Ratna Rao ${ }^{7}$ \\ ${ }^{1}$ Department of Microbiology, Prathima Institute of Medical Sciences, Karimnagar, India \\ ${ }^{2}$ Department of Biochemistry, Vydehi Institute of Medical Sciences and Research Centre, Bangalore, India \\ ${ }^{3}$ Department of Dermatology, Vydehi Institute of Medical Sciences and Research Centre, Bangalore, India \\ ${ }^{4}$ Department of Biochemistry, Akash Institute of Medical Sciences \& Research Center, Bangalore, India \\ ${ }^{5}$ Department of Biochemistry, Chalmeda Anandarao Institute of Medical Sciences, Karimnagar, India \\ ${ }^{6}$ Department of Microbiology, Prime Hospitals, Hyderabad, India \\ ${ }^{7}$ Department of Microbiology, Apollo Hospitals, Jubilee Hills, Hyderabad, India \\ *Corresponding author: ramana_20021@rediffmail.com
}

Received February 26, 2013; Revised December 06, 2013; Accepted December 24, 2013

\begin{abstract}
Complicated skin and skin structure infections (cSSSI's) are among the most common microbial infections occurring both in the community and the hospitals. Aetiology of cSSSI's is complex and includes various microorganisms (bacteria, fungi, parasites and viruses). cSSSI's in general are poly microbial in nature including both anaerobic and aerobic bacterial species. Primary cSSSI's resulting from single bacterial species and secondary bacterial infections in patients suffering from skin conditions due to autoimmune conditions (eczema) or other microbial causes (viruses, fungi and parasites) have been reported in the literature. Occurrence of multi-drug resistant bacteria, underlying co-morbidities contribute to the complications in the management of cSSSI's. Formulating effective guidelines for clinical, laboratory diagnosis, treatment and management of patients suffering from cSSSI's would certainly be beneficial in the reduction of morbidity and mortality.
\end{abstract}

Keywords: complicated skin and skin structure infections (cSSSI's), aetiology of cSSSI's, multi-drug resistance

Cite This Article: K V Ramana, Venkata BharatKumar Pinnelli, Bhanu Prakash, Wilma Delphine Silvia CR, Sabitha Kandi, CH V Sharada, Anand Kalaskar, Sanjeev D Rao, Ratna Mani, and Ratna Rao, "Complicated Skin and Skin Structure Infections (cSSSI's): A Comprehensive Review." American Journal of Medical and Biological Research 1, no. 4 (2013): 159-164. doi: 10.12691/ajmbr-1-4-9.

\section{Introduction}

Complicated skin and skin structure infections (cSSSI's) constitute microbial infections of skin and soft tissues. cSSSI's may usually present as a mild superficial and suppurative skin infection (pyoderma) and can later be developing in to severe necrotizing fasciitis involving subcutaneous connective tissues and spread deep in to other soft tissues [1]. cSSSI's are a cause of huge public health problem resulting in both community acquired infections as well as nosocomial infections [2]. In the era of HIV and emergence of various infectious agents and malignancies, that may be responsible for compromised immune status of individuals, cSSSI's can result in greater morbidity and mortality especially in the developing world. Prompt clinical and laboratory diagnosis and initiation of effective antimicrobial therapy remains mainstay in the management of cSSSI patients. Prevalence of multi drug resistance and associated co-morbidities including diabetes, chronic kidney diseases, liver dysfunction, cardiovascular diseases, viral infections (HIV, Hepatitis B virus, Hepatitis C virus and others), cancerous conditions and age of the patient influence the prognosis of cSSSI's [3]. Studies have also noted that military personnel with war wounds are predisposed to cSSSI's. The nature of infections in case of cSSSI's has been confined to the area of inoculation and local spread to deep tissues, but dissemination hematogenously causing septicemia has also been reported [3]. Animal bite/scratch wounds may also be responsible for skin and skin structure infections (SSSI's) [4]. Predisposing factors that contribute to the development of cSSSI's include peripheral vascular insufficiency, peripheral neuropathy secondary to diabetes/cardiovascular diseases, disrupted venous or lymphatic circulatory system, inoculation of foreign bodies (war wounds, accident trauma), poor hygiene and immunocompromised conditions (chronic renal failure, steroid use, immunosuppressive therapy, organ transplantation HIV infection) [5,6]. Classification of cSSSI's is complex and depends on various clinical and laboratory paramerters. cSSSI's can be superficial, deep or disseminating hematogenously depending on the extent of invasion (superficial skin, sub cutis, fascia and muscle tissue). They can be either necrotizing or non-necrotizing and acute or chronic based on the presentation where acute infections usually take few days to weeks for resolving and chronic infections may take months to resolve. cSSSI's can also be defined as those that require 
emergency surgical attention and those that can be managed medically with antimicrobial therapy. Uncomplicated SSSI's present as cellulitis, impetigo, erysipelas, carbuncles, furuncles and superficial abscess. Complicated SSSI's usually present as deep tissue infection, spreading and necrotizing cellulitis, infected ulcer, burns wound and deep tissue abscesses Figure 1 and Figure 2. Infections of skin and its structures attributed to prosthetic devices, rare immunodeficiency and hypersensitivity conditions (atopy) are not included under cSSSI's $[7,8]$. In the era of extensive dissemination of multi-drug resistant bacterial species, presence of Extended spectrum beta lactamases (ESBL), Methicillin resistant Staphylococcus aureus (MRSA), Vancomycin resistant Staphylococcus aureus, vancomycin and Glycopeptide resistant enterococci, the clinical, laboratory diagnosis and management of cSSSI's assume greater significance [9,10,11,12]. Although most cSSSI's are uncomplicated, guidelines for better management result in reduced morbidity and mortality [13,14]. It has been reported that SSSI's mimic other conditions like the deep vein thrombosis, thrombophlebitis, gout, carcinoma erysipeloides, adverse drug reaction and foreign body reaction $[15,16]$.

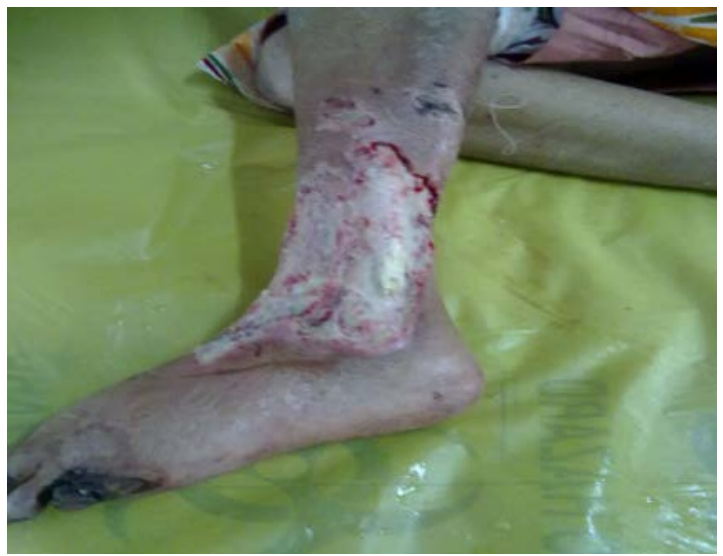

Figure 1. complicated skin and skin structure infection of leg spreading and deep in to the muscle exposing the skeleton with necrosis of toe

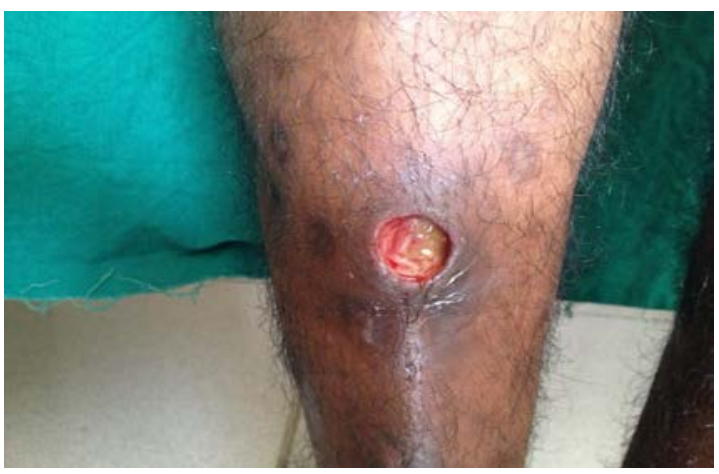

Figure 2. Deep abscess of the leg below knee

\section{Aetiology of Complicated Skin and Skin Structure Infectionse}

In contrast to the other superficial skin infections the etiology of cSSSI's usually is polymicrobial in nature including anaerobic bacteria. Potential causes for community acquired cSSSI's include Staphylococcus aureus, group A Streptococci. Gram negative bacterial causes include non-fermenters like Pseudomonas aeruginosa, Acinetobacter spp and others, which are usually associated with nosocomial CSSI's [5]. Ellie J C Goldstein et al in their study have revealed that 33.5\% of cSSSI's were polymicrobial in nature with abscess, diabetic foot ulcer and post traumatic wound ulcers accounting for most of the cSSSI's. the same study has shown that gram positive bacteria including Staphylococcus aureus were responsible for $24 \%$ of infections and 53\% of cSSSI's were attributed to gram negative bacteria including the anaerobes (Bacteroides fragilis, Prevotella spp, Pophyromonas spp, Fusobacterium spp, Veillonella spp and others. Among the anaerobic bacterial causes, $34.9 \%$ of the cSSSI's were showing Peptostreptococcus spp [17]. Recent studies have also noted the occurrence of monomicrobial skin and skin structure infections (SSSI's) involving Acinetobacter baumannii [18]. The same study has elaborated on the possible co-morbidities including severe physical trauma and liver cirrhosis, chronic kidney disease, obesity, old age and nosocomial infection associated with another pathogen, diabetes and cancer, which may complicate the management of SSSI's [18]. It has also been noted that enterobacteriaceae members including Escherechia coli, Klebsiella pneumoniae and other bacterial species of gastrointestinal origin like Enterococcus faecalis may be responsible for both complicated and uncomplicated SSSI's [6]. Other bacteria that have been associated with cSSSI's include Vibrio vulnificus, Aeromonas hydrophila, Clostridium spp and group B, C, G Strptococcus spp and other microaerophilic bacterial species. Haemophilus influenza, Bacillus anthracis, pasteurella multocida, Pasteurella spp, Erysipelothrix rhusiopathiae and other vibrio spp including Vibrio carchariae, Vibrio damsela, Vibrio hollisae and vibrio alginolyticus have been noted causes of cSSSI's Table 1 [4,6]. A recent study from Spain by Raya-Cruz $M$ et al have evaluated skin and soft tissue infections in hospitalized patients and found that $66.7 \%$ infected patients presented with cellulitis/erysipelas and diabetes (33\%), heart failure $(17.7 \%)$ were found to be frequent co-morbidities. The same study has revealed that Staphylococcus aureus (35.1\%) and MRSA(12.9\%) were most common causes [19]. A recent study from India by Phakade RS et al has revealed that Staphylococcus aureus (73\%) and streptococcus spp (12\%) were frequent Gram positive causes for community acquired skin and soft tissue infections where as Pseudomonas aeruginosa (28\%) and Acinetobacter spp (18\%) were associated with hospital acquired SSI's [20]. Skin, being the largest organ of the body present outside as a protection to the internal organs is most prone to external injuries. Skin and its underlying fatty layer, fascia, muscle is a tough and flexible structure that may be prone to infection/inflammation. Skin infections are best described way back in $1^{\text {st }}$ century as calor, rubor, tumor, dolor, and fluor indicating heat, redness, swelling, pain and discharge respectively [3,21]. Skin consists of normal microbial flora including gram positive cocci, yeasts and other bacterial species, which protect it from invasion of potential pathogens. Trauma and breakage in this stricture may predispose the skin to have given access to colonization and invasion of pathogenic microorganisms. 
Table 1. Aetiology and predisposing factors contributing to complicated skin and skin structure infections (cSSSI's)

\begin{tabular}{|c|c|}
\hline ( & Predisposing factors \\
\hline Common Bacterial causes & Diabetes \\
\hline Staphylococcus aureus & Long term Streoid therapy \\
\hline Coagulase negative Staphylococci (CONS) & Neutropenia \\
\hline Streptococcus pyogenes & Liver cirrhosis \\
\hline Streptococcus spp (Group B, C, G and H) & Burns \\
\hline Enterococcus spp & Alcoholism \\
\hline Pseudomonas spp & Organ transplantation \\
\hline Acinetobacter spp & Malnutrition \\
\hline Citrobacter spp & Infectious disease (HIV and others) \\
\hline Enterobacteriaceae members (Escherechia coli, Klebsiella spp, Proteus & Extremes of age \\
\hline spp, Enterobacter spp, Serretia spp, Salmonella spp and others) & Long duration hospitalization \\
\hline Vibrio spp & Autoimmune diseases \\
\hline Aeromonas hydrophila & \\
\hline Mycobacterium spp & \\
\hline Clostridium spp & \\
\hline Haemophillus influenzae & \\
\hline Human and Animal bite/contact/ wounds & \\
\hline Pasteurella multocida & \\
\hline Pasteurella spp & \\
\hline Campylobacter spp & \\
\hline Capnocytophaga canimorsus & \\
\hline Streptobacillus moniliformis & \\
\hline Bartonella henselae & \\
\hline Fransisella tularensis & \\
\hline Bacillus anthracis & \\
\hline Yersinia pestis & \\
\hline Spirullum minor & \\
\hline CDC group EF4 & \\
\hline Vibrio carchariae & \\
\hline Fusobacterium spp & \\
\hline Prevotella spp & \\
\hline Unusual bacterial causes & \\
\hline Vibrio fluvialis & \\
\hline Vibrio vulnificus & \\
\hline Eikenella corrodens & \\
\hline Nocardia spp (soil contamination) & \\
\hline Erysipelothrix rhusiopathiae & \\
\hline Helicobacter cinaedi & \\
\hline Infectious diseases presenting as Skin lesions & \\
\hline Neisseria meningitidis & \\
\hline Streptococcus pyogenes (scarlet fever) & \\
\hline Rickettsial infections & \\
\hline Treponema pallidum (syphilis) & \\
\hline Candida spp (Tinea) & \\
\hline Cryptococcus spp (Cutaneous) & \\
\hline Systemic fungal diseases & \\
\hline Ancylostoma duodenale ( cutaneous larva migrans) & \\
\hline Leishmania spp & \\
\hline Trypanosoma spp & \\
\hline Measles & \\
\hline Varicella zoster virus & \\
\hline Herpes virus & \\
\hline Human Immunodeficiency Virus & \\
\hline
\end{tabular}

\section{Antimicrobial Therapy and Newer Therapeutic Approaches in cSSSI's}

Complicated SSSI's are among the most common infectious conditions in human that warrant antimicrobial therapy. As most cSSSI's involve more than one type of bacteria including the anaerobic bacterial species, choice of antimicrobials selected as empirical therapy must be having broad spectrum of activity. Empirical therapy should be formulated keeping in mind the frequency of multi-drug resistant bacteria, the beta-lactamse, Extended spectrum beta-lactamase (ESBL) and cabapenemase/metallo-beta-lactamase (MBL) producing bacterial species. A previous study that included patients suffering from cSSSI's has noted that etrapenem and piperacillin-tazobactum were effective against more than 97\% of bacterial species (anerobic and aerobic bacteria)
[17]. Having noted the frequent occurrence of MRSA and other multi-drug resistant gram positive bacteria, a study noted that telavancin, a newer injectible drug, a lipoglycopeptide having bactericidal activity was found effective against MRSA, vancomycin intermediate susceptible and vancomycin resistant gram positive bacterial isolates [22]. Another recent study that has evaluated the efficacy of telavancin and vancomycin against gram positive bacterial isolates of cSSSI's has revealed that telavancin was indeed more effective (93\%) than vancomycin (90\%) against Panton-Valentine leucocidin (PVL) positive MRSA isolates [23]. In the era of multi-drug resistance, there is an argument on the cautious use of antimicrobial agents to reduce the possibility of development of resistance [24]. A study that has reviewed a decade long data on the incidences of cSSSI's, the nature of therapy and the outcome of treatment has shown that the average cure rate among those who were not given antimicrobial therapy was $66 \%$ 
as compared to those who were administered systemic antibiotics that included sulfonamides (91\%) and penicillin (98\%) group drugs [25]. Previous studies have also noted that topical antimicrobial therapy was not as effective as intravenous penicillin/sulfonamide therapy in the treatment of cSSSI's [26]. Having noted the prevalence of multi-drug resistant gram positive bacterial involvement in cSSSI's, a previous study has revealed the efficacy of daptomycin, a cyclic lipopeptide antimicrobial agent against vancomycin intermediate susceptible/ resistant, methiciliin resistant and MDR strains of Enterococcus spp and Staphycoccus spp [27]. Comparative efficacy of newer and injectable streptogramin antimicrobial agent, the Quinupristin/dalfopristin (synercid) against conventional therapeutic agents (cefazolin, oxacillin, vancomycin) revealed that with regards to the clinical success rate both therapeutic strategies showed similar results. This study has also noted that bacteriological eradication rates were lower in Quinupristin/dalfopristin group (68\%) than in conventional group (76\%) and that drug related toxicity was higher in Quinupristin/dalfopristin group (66\%) when compared with conventional group (28\%) [28]. Activity of a newer cephalosporin, ceftaroline against both multidrug resistant gram positive bacteria and gram negative bacteria in cSSSI's has been emphasized in a recent papers [29,30]. Novel antimicrobial combination therapy using colistinrifampicin and carbapenem-sulbactum group have been found effective in the treatment of MDR strains of Acinetobacter baumannii [31,32]. A review article published recently has illustrated the activity (bactericidal/bacteriostatic) of preferred antimicrobial agents (linazolid, tigecycline, daptomycin, vancomycin, TMP-SMX, rifampicin, fosfomycin, clindamycin and quinupristin/dalfopristin) in the treatment of cSSSI's and revealed that eradicate rate was highest (85\%) among those treated with intravenous/oral linazolid [33,34]. Meta-analysis study performed on the efficacy of tigecycline in the treatment of cSSSI's and other infectious diseases has revealed that tigecycline was not inferior (though some adverse drug reactions were noted) to vancomycin and other conventional therapeutic modalities in the management of cSSSI's [35]. Efficacy of tigecycline in the treatment of complicated infections in hospitalized patients was evaluated in a prospective, multicenter and non-intervention study by Macus J Zervos et al and found that clinical cure rate for the treatment of cSSSI's was found to be $82 \%$. Results from a recent randomized clinical trial has revealed that treatment with intravenous/oral moxifloxacin followed by oral amoxicillin-clavulinic acid was found both clinically and bacteriologically as effective as intravenous piperacillintazobactum in the treatment of cSSSI's [36]. Monotherapy with moxifloxacin (oral/Intravenous) was found to be very effective and safe in the improvement and resolution of cSSSI's in a recent observational study [37]. A recent study from America has reviewed novel therapies for the treatment and management of skin infections caused by Acinetobacter baumannii [38]. This paper suggested that being an opportunistic bacteria that is prevalent in the hospitals and having developed resistance to multiple drugs including production of the ESBL's and MBL's, therapeutic approach for the treatment of skin infections caused by A baumanni and other multi-drug resistant bacteria include bactericidal gene transfer therapy (introduction of plasmid containing bactericidal genes in to the pathogenic bacteria), bioengineering human skin tissue (human skin is bioengineered to produce nontumorogenic, pathogen free human keratinocyte progenitor cells (NIKS) capable of synthesizing host defence peptides such as cathecidins and defensins that help in wound healing), Nitic-oxide releasing nanoparticles (nano-technology mediated delivery of niticoxide nanoparticles in to the affected/infected skin is used to enhance wound healing), phage therapy (multi-drug resistant bacteria are cytolysed by infecting them with specific bacteriophages), photodynamic therapy (PDT) (use of non-toxic photosensitizers (PS's) and harmless visible light, that in the presence of oxygen, generate reactive oxygen species that eliminate cancerous cells and kill infectious microorganisms) and radio immunotherapy (RIT) (a method where in the affected organ is exposed to radioactive material that is toxic and cytolytic in nature) [39-48]. Another approach to the treatment and management of complicated SSSI's with MDR bacteria is named as unconventional therapy, where the antimicrobial agent is given in combination with a plant extract that act synergistically in the elimination of infection [49]. Utility of blue light (light wavelength of $415 \pm 10 \mathrm{~nm}$ ) as a therapeutic option for the elimination of skin infections caused by MRSA was studied in experimental mice by Tianhong Dai et al. This study showed that blue light was effective in rapidly reducing the burden of bacteria from the infection site [49]. Peptide antibiotics/anti infective agents which are considered as next generation therapeutic agents may help in the treatment and management of cSSSI's in future [51].

\section{Management of Complicated SSSI's}

A recent study by Wilson et al, developed a severity-ofillness scoring system for the better management of cSSSI's based on the resultant clinical cure rates. This was later reviewed for its utility in scoring the disease and was found not suitable in predicting the clinical outcome as these studies were not addressing the role of comorbidities, epidemiological variability's, demographical influences and clinical approaches [52,53]. In a recent retrospective observational cohort study by Garau et al that performed syatematic analysis of data on management practices of cSSSI's collected from 129 centers in ten European countries, revealed that of the 1995 patients included in the study the mean age was 60 years. The study performed a review of disease characteristics, treatment modalities and clinical outcome. The results of the study revealed that $40 \%$ of the patients needed initial therapy change which was influenced by the comorbidities (42.6\%) and surgical procedure (44\%). This study also showed that the mortality rate was found to be $3.4 \%$ and the mean hospital stay was $18 \pm 19.9$ days [54]. Management of cSSSI's has been noted to be influenced by the nature of the clinical infection and its severity in to three classes (Class 1, Class 2, class 3, class 4). Classes 1 are all those patients with no systemic signs of toxicity and co-morbidities, class 2 patients include those who are systemically un well and have any one co-morbidity, class 3 includes patients who are clinically defined as showing 
toxic signs and patients suffering from extensive necrotizing lesions are placed in class [4,55]. Previous studies have suggested that depending on the severity, cSSSI's can be classified as un-complicated (impetigo, ecthyma, erysipelas, folliculitis, furanculosis and other superficial focal skin infections), complicated infections (secondary infections of diseased skin (autoimmune/infectious conditions), cellulitis, lymphangitis, carbuncles, traumatic, bite wounds and others) and soft tissue infections (peri-anal abscess, diabetic wound infections, necrotizing soft tissue infections, Fournier's gangrene, myonecrosis, clostridia/traumatic/atraumatic gas gangrene). Most SSSI's are uncomplicated and require no hospital admission in contrast to the complicated SSSI's that necessitate hospital stay and close monitoring. Management of cSSSI's require immediate attention on the extent of skin leision (involving muscle/fascia), signs of toxemia, necessity for surgical intervention, diagnostic tests to be performed and the empirical antimicrobial therapeutic options [4,6,13]. After a thorough clinical evaluation and on making specific decision on the further management of patients either without surgical intervention (antimicrobial therapy-topical/intravenous) or having decided on surgical procedures (debridement/plastic surgery), the clinicians should consider laboratory evaluation for the possible aetiology and antimicrobial susceptibility patterns of the isolated infectious causes. Initiation of a broad spectrum antimicrobial empirical therapy (topical/systemic), or deciding on therapeutic options after the availability of antimicrobial susceptibility reports would be beneficial in better patient management of cSSSI's.

\section{Conclusions and Future Perspectives}

In the era of increasing antimicrobial resistance and occurrence of multi-drug resistant bacteria (MDR), studies have now been concentrating on the development of novel therapeutic peptide antimicrobial agents [56,57]. Patient management be made on careful clinical considerations, reducing the time to make a decision on how to manage (therapeutic only/need for surgical intervention), utilizing relevant and reliable laboratory diagnostic methods and initiating appropriate antimicrobial therapy [58]. Developing and poor third world nations will have to formulate effective management strategies for patients suffering from cSSSI's considering the fact that there is a huge cost of hospitalization and antimicrobial therapy associated with the treatment of cSSSI's.

\section{References}

[1] Rhody, C. Bacterial infections of the skin. Primary Care 2000; 27: 459-73.

[2] Zilberberg MD, Shorr AF, Micek ST, et al. Hospitalizations with healthcare-associated complicated skin and skin structure infections: impact of inappropriate empiric therapy on outcomes. J Hosp Med 2010; 5:535-540.

[3] Mathew S Dryden. Complicated skin and soft-tissue infection. J Antimicrob Chemother 2010; 65 Suppl S3: iii 35-44.

[4] Mark J. DiNubile and Benjamin A. Lipsky. Complicated infections of skin and skin structures: when the infection is more than skin deep. J Antimicrob Chemother 2004; 53 Suppl S2: ii37ii 50 .
[5] P Kujath and C Kujath. Complicated skin, skin structure and softtissue infections -Are we threatened with multi-resistant pathogens? Eur J Med Res 2010; 15: 544-553.

[6] Sabitha Rajan. Skin and soft-tissue infections: classifying and treating a spectrum. Claveland Clinic Journal of Medicine 2012; 79 (1): 57-66.

[7] James I. Merlino and Mark A Malangoni. Complicated skin and soft-tissue infections: Diagnostic approach and empiric treatment options. Claveland Clinic Journal of Medicine 2007; 74 (supplement 4): S 21-S 28.

[8] Cornia PB, Davidson HL, Lipsky BA. The evaluation and treatment of complicated skin and skin structure infections. Expert Opin Pharmacother 2008; 9:717-730.

[9] Kalaskar A, Venkataramana K. Determination of Antimicrobial Resistance Pattern and Production of Extended-Spectrum $\beta$ Lactamases amongst Escherichia coli and Klebsiella pneumoniae from Clinical Isolates. J Med Bacteriol. 2012; 1 (2): pp. 17-24.

[10] Ramana KV, Mohanty SK, Kumar A. In-vitro activities of current antimicrobial agents against isolates of pyoderma. Indian $\mathrm{J}$ Dermatol Venereol Leprol 2008; 74:430.

[11] Ramana KV, Mohanty SK, Wilson CG. Staphylococcus aureus colonization of anterior nares of school going children. Indian $\mathrm{J}$ Pediatr. 2009 Aug; 76(8):813-6.

[12] Ramana KV and Rao R. Significance of Screening for Colonization and Vancomycin Resistance in Staphylococcus aureus Isolated from Anterior Nares of School Going Children. Online J Health Allied Scs. 2009; 8(3):20.

[13] Stevens DL, Bisno AL, Chambers HF, et al. Practice guidelines for the diagnosis and management of skin and soft-tissue infections. Clin Infect Dis 2005; 41:1373-1406.

[14] Center for Drug Evaluation and Research, Food and Drug Administration, U.S. Department of Health and Human Services. Guidance for industry [draft]. Uncomplicated and complicated skin and skin structure infections developing antimicrobial drugs for treatment. July $1998 . \quad$ Available at: http://www.fda.gov/Cder/guidance/2566dft.pdf. $\quad$ Accessed November 6, 2013.

[15] Papadavid E, Dalamaga M, Stavrianeas N, Papiris SA. Subcutaneous sarcoidosis masquerading as cellulitis. Dermatology 2008; 217:212-214.

[16] Falagas ME, Vergidis PI. Narrative review: diseases that masquerade as infectious cellulitis. Ann Intern Med 2005; 142:4755.

[17] Ellie J. C. Goldstein, Diane M. Citron, C. Vreni Merriam, Yumi Warren, Kerin L. Tyrrell, and Richard M. Gesse. General Microbiology and In Vitro Susceptibility of Anaerobes Isolated from Complicated Skin and Skin-Structure Infections in Patients Enrolled in a Comparative Trial of Ertapenem Versus PiperacillinTazobactam. Clin Infect Dis 2002; 35 (suppl 1): S 119-25.

[18] Lee NY, Wang CL, Chuang YC, Yu WL, Lee HC, Chang CM, et al. Combination carbapenem-sulbactam therapy for critically ill patients with multidrug-resistant Acinetobacter baumannii bacteremia: four case reports and an in vitro combination synergy study. Pharmacotherapy 2007; 27:1506-11.

[19] Raya-Cruz M, Ferullo I, Arrizabalaga-Asenjo M, Nadal-Nadal A, Díaz-Antolín MP, Garau-Colom M, Payeras-Cifre A. Skin and soft-tissue infections in hospitalized patients: Epidemiology, microbiological, clinical and prognostic factors. Enferm Infecc Microbiol Clin. 2013 May 15. pii: S 0213-005 X (13)00077-3.

[20] Phakade RS, Nataraj G, Kuyare SS, Khopkar US, Mehta PR. Is methicillin-resistant Staphylococcus aureus involved in community acquired skin and soft tissue infections? Experience from a tertiary care centre in Mumbai. J Postgrad Med. 2012 JanMar; 58(1):3-7.

[21] Mims C, Playfair J, Roitt I et al. Medical Microbiology. Mosby Int Ltd, London.

[22] Lala M Dunbar, Derek M Tang and Robert M Manausa. A review of telavancin in the treatment of complicated skin and skin structure infections (cSSSI's). Therapeutics and Clinical Risk Management 2008; 4 (1): 235-244.

[23] Martin E. Stryjewski, Steven L. Barriere, William O’Riordan, Lala M. Dunbar, Alan Hopkins, Fredric C. Genter and G. Ralph Corey. Efficacy of telavancin in patients with specific types of complicated skin and skin structure infections J Antimicob Chemother 2012; 67: 1496-1502.

[24] Ramana KV, Rao R, Sharada C, Kareem MA, Reddy LR, Ratna Mani MS. Modified Hodge test: A useful and the low cost 
Phenotypic method for detection of carbapenemase producers in Enterobacteriaceae members. J Nat Sc Biol Med 2013; 4:346-8.

[25] Brad Spellberg, George H. Talbot, Helen W. Boucher, John S. Bradley, David Gilbert, W Michael Scheld, John Edwards Jr. and John G. Bartlett for the Antimicrobial Availability Task Force of the Infectious Diseases Society of America. Antimicrobial Agents for Complicated Skin and Skin-Structure Infections: Justification of Noninferiority Margins in the Absence of Placebo-Controlled Trials Clin Infect Dis 2009; 49 (3): 383-391.

[26] Keefer CS, Blake FG, Marshall EK Jr, Lockwood JS, Wood BW. Penicillin in the treament of infections. JAMA 1943; 122:1217-24.

[27] Judith N. Steenbergen, Jeff Alder, Grace M. Thorne and Francis P. Tally. Daptomycin: a lipopeptide antibiotic for the treatment of serious Gram-positive infections. J Antimicob Chemother 2005; 55: 283-288.

[28] Ronald L Nichols, Donald R Graham, Steven L Barriere, Anthony Rodgers, Samuel E Wilson, Marcus Zervos, David L Dunn and Bruce Kreter for the synercid skin and skin structure infection group. J Antimicob Chemother 1999; 44: 263-273.

[29] Donald Biek, Ian A. Critchley, Todd A. Riccobene and Dirk A. The Ceftaroline fosamil: a novel broad spectrum cephalosporin with expanded anti-Gram-positive activity. J Antimicrob Chemother 2010; 65 Suppl 4: iv9-16.

[30] Robert C. Moellering Jr. The problem of complicated skin and skin structure infections: the need for new agents. J Antimicrob Chemother 2010; 65 Suppl 4: iv3-8.

[31] Bassetti M, Repetto E, Righi E, Boni S, Diverio M, Molinari MP, et al. Colistin and rifampicin in the treatment of multidrugresistant Acinetobacter baumannii infections. J Antimicrob Chemother 2008; 61:417-20.

[32] Dubert M. Guerrero, Federico Perez, Mark D. Adams, Nicholas G. Conger, Philip N. Rather, Joseph S. Solomkin, and Robert A. Bonomo. Acinetobacter baumannii-Associated Skin and Soft Tissue Infections: Recognizing a Broadening Spectrum of Disease. Surg Infect 2011; 11 (1): 49-57.

[33] Christian Eckmann and Mathew Dryden. Treatment of Complicated skin and soft-tissue infections caused by resistant bacteria: value of linazolid, tigecycline, daptomycin and vancomycin. Eur J Med Res 2010; 15; 554-563.

[34] Beth White and $\mathrm{R}$ Andrew Seaton. Complicated skin and soft tissue infections: Literature review of evidence for and experience with daptomycin. Infection and Drug Resistance 2011; 4: 115-127.

[35] Yun Cai, Rui Wang, Beibei Liang, Nan Bai and Youning Liu. Systematic Review and Meta-Analysis of the Effectiveness and Safety of Tigecycline for Treatment of Infectious disease. Antimicrobial agents and Chemotherapy 2011; 55 (3): 1162-1172.

[36] Inge C. Gyssens, Matthew Dryden, Peter Kujath, Dilip Nathwani, Nicolaas Schaper, Barbara Hampel, Peter Reimnitz, Jeff Alder and Pierre Alvis. A randomized trial of the efficacy and safety of sequential intravenous/oral moxifloxacin monotherapy versus intravenous piperacillin/tazobactam followed by oral amoxicillin/clavulanate for complicated skin and skin structure infections. J Antimicrob Chemother 2011; 66: 2632-2642.

[37] Bogner JR, Kutaiman A, Esguerra-Alcalen M, Heldner S, Arvis P. Moxifloxacin in complicated skin and skin structure infections (cSSSIs): A prospective, international, non-interventional, observational study. Adv Ther. 2013 Jun; 30(6):630-43.

[38] Mircea Radu Mihu and Luis R Martinez. Novel therapies for treatment of multidrug resistant Acinetobacter baumannii skin infections. Virulence 2011; 2 (2): 97-102.

[39] Shankar R, He LK, Szilagyi A, Muthu K, Gamelli RL, Filutowicz $\mathrm{M}$, et al. A novel antibacterial gene transfer treatment for multidrug resistant Acinetobacter baumannii-induced burn sepsis. J Burn Care Res 2007; 28:6-12.

[40] Thomas-V irnig CL, Centanni JM, Johnston CE, He LK, Schlosser SJ, Van Winkle KF, et al. Inhibition of multidrug-resistant Acinetobacter baumannii by nonviral expression of hCAP-18 in a bioengineered human skin tissue. Mol Ther 2009; 17:562-9.

[41] Heilborn JD, Nilsson MF, Kratz G, Weber G, Sørensen O, Borregaard N, et al. The cathelicidin anti-microbial peptide LL-37 is involved in re-epithelialization of human skin wounds and is lacking in chronic ulcer epithelium. J Invest Dermatol 2003; 120:37.

[42] Mowbray M, Tan X, Wheatley PS, Rossi AG, Morris RE, Welle RB. Topically applied nitric oxide induces T-lymphocyte infiltration in human skin, but minimal inflammation. J Invest Dermatol 2008; 128:352-60

[43] Soneja A, Drews M, Malinski T. Role of nitric oxide, nitroxidative and oxidative stress in wound healing. Pharmacol Rep 2005; 57:108-19.

[44] Friedman AJ, Han G, Navati MS, Chacko M, Gunther L, Alfieri A et al. Sustained release nitric oxide releasing nanoparticles: Characterization of a novel delivery platform based on nitrite containing hydrogel/glass composites. Nitric Oxide 2008; 19:1220.

[45] Lin NT, Chiou PY, Chang KC, Chen LK, Lai MJ. Isolation and characterization of phi AB2: a novel bacteriophage of Acinetobacter baumannii. Res Microbiol 2010; 161:308-14.

[46] Castano AP, Mroz P, Wu MX, Hamblin MR. Photodynamic therapy plus low-dose cyclophosphamide generates antitumor immunity in a mouse model. Proc Natl Acad Sci USA 2008; 105:5495-500.

[47] Dadachova E, Casadevall A. Antibodies as delivery vehicles for radioimmunotherapy of infectious diseases. Expert Opin Drug Deliv 2005; 2:1075-84. Radioimmunotherapy.

[48] Dadachova E, Burns T, Bryan RA, Apostolidis C, Brechbiel MW, Nosanchuk JD, et al. Feasibility of radioimmunotherapy of experimental pneumococcal infection. Antimicrob Agents Chemother 2004; 48:1624-9.

[49] Wang HM, Chen Cy, Chen HA, Huang WC, Lin WR, Chen TC, et al. Zingiber officinale (ginger) compounds have tetracyclineresistance modifying effects against clinical extensively drugresistant Acinetobacter baumannii. Phytother Res 2010; 24:182530 .

[50] Tianhong Dai, Asheesh Gupta, Ying-Ying Huang, Margaret E. Sherwood, Clinton K. Murray, Mark S. Vrahas, Tammy Kielian and Michael R. Hamblin Blue Light Eliminates CommunityAcquired Methicillin-Resistant Staphylococcus aureus in Infected Mouse Skin Abrasions. Photomedicine and Laser Surjery 2013;31 (11): 531-538.

[51] Derry K Mercer \& Deborah A O’Neil. Peptides as the next generation of anti-infectives. Future Medicinal Chemistry 2013, Vol. 5, No. 3, Pages 315-337.

[52] Wilson SE, Solomkin JS, Le V, Cammarata SK, Bruss JB. A severity score for complicated skin and soft tissue infections derived from phase III studies of linezolid. Am. J. Surg. 2003; 85:369-375.

[53] George H. Talbot, Tanya O’Neal, Anita F. Das, Dirk Thye. Prospective Study of the Wilson Severity-of-Illness Scoring System for Complicated Skin and Skin Structure Infections. Antimicrobial Agents and Chemotherapy 2013; 57 (1): 647-650.

[54] Garau, J.; Ostermann, H.; Medina, J.; et al. Current management of patients hospitalized with complicated skin and soft tissue infections across Europe (2010-2011): assessment of clinical practice patterns and real-life effectiveness of antibiotics from the REACH study. CLINICAL MICROBIOLOGY AND INFECTION Volume: 19 Issue: 9 Pages: E 377-E 385.

[55] Eron LJ, Lipsky BA, Low DE et al., Managing skin and soft tissue infections: Expert panel recommendations on key decision points. J Antimicrob Chemother 2003; 52 Suppl 1: i3-17.

[56] Fernando Albericio and Hendrik G Kruger. Therapeutic Peptides. Future Med Chem 2012; 4 (12): 1527-1531.

[57] Jenkins TC, Knepper BC, Sabel AL, et al. Decreased antibiotic utilization after implementation of a guideline for inpatient cellulitis and cutaneous abscess. Arch Intern Med 2011; 171:10721079 .

[58] Hammond SP, Baden LR. Clinical decisions. Management of skin and soft-tissue infection-polling results. N Engl J Med 2008; 359 e 20 . 